\title{
Elastic exchange scattering amplitude studied using magnetic EXAFS
}

\author{
D. Ahlers and G. Schütz \\ Universität Augsburg, Institut für Experimentalphysik II, Memmingerstraße 6, D-86135 Augsburg, Germany
}

(Received 25 August 1997)

\begin{abstract}
Magnetic or spin-dependent extended x-ray-absorption fine-structure (EXAFS) studies have been performed at the $K$ edges of pure $\mathrm{Co}$ and $\mathrm{Ni}$ and at the $L_{1,2,3}$ edges of $\mathrm{Gd}$ metal. Using a two-step model for the absorption process and introducing an additive-exchange contribution to the complex Coulomb scattering amplitude and the electron mean free path, an ansatz for the description of the magnetic EXAFS is derived. A parametrization of these quantities is proposed for an extraction of the spin-dependent values from the experimental data. The results for the elastic exchange scattering cross section in the energy range of 100-600 eV are in excellent agreement with theoretical predictions from a Born-Ockhur approximation. The inelastic exchange scattering effects are found to be negligible. [S0163-1829(98)01906-7]
\end{abstract}

\section{INTRODUCTION}

Since the last decade the study of $\mathrm{x}$-ray magnetic circular dichroism (XMCD) in near-edge core-level absorption has become a powerful tool to get element-specific insight into the ferro(i)magnetism of solids on an atomic or microscopic scale. As already observed in the pioneering XMCD measurements, even in the extended $\mathrm{x}$-ray-absorption finestructure (EXAFS) range the existence of a magnetic contribution is found to be a universal phenomenon. ${ }^{1}$ Meanwhile this spectroscopic method is expected to provide new attractive possibilities to study the magnetic short-range order in ferro(i)magnetic materials. ${ }^{2-4}$ One of the most important aspects concerning the structural information is the possibility of distinguishing the electronic and magnetic neighborhood of the absorbing atom by comparison of the EXAFS and spin-dependent (SP) EXAFS. ${ }^{5,6}$ From a variety of systematic studies the relative amplitude of the dichroic contribution is found to be directly proportional to the average number of spin-polarized electrons of the first coordination shell, thus allowing a quantitative determination of the next-neighbor spin moment.

More recently a variety of attempts to formulate a complete description of the experimental data have been performed. $^{7-9}$ These theories are based on the multiplescattering expansion formalism commonly applied to normal EXAFS. The atomic potentials in the EXAFS case are generally assumed to be independent of the spin and spherically symmetric, i.e., independent of the magnetic quantum number $m_{l}$. This simplification is not justified in case of ferromagnetically ordered systems. The aim of the theory is therefore to calculate accurate spin-dependent potentials and to include the scattering from asymmetric potentials in the multiple-scattering expansion. In case of the $\mathrm{Gd} L_{2,3}$ edge theoretical calculations of spin-dependent EXAFS were published recently by Ankudinov and Rehr. ${ }^{7}$ Here the half-filled shell of Gd ensures that the spherical symmetry of the scattering potential is retained. For the $l=1$ initial states it is suggested that the spin-orbit interaction only for the core electrons has to be taken into account, whereas it is neglected for the photoelectron. This procedure, however, makes it very difficult to access more complex magnetic systems and the magnetic quantities of interest. A special case is the $K$ and $L_{1}$ absorption, where, despite the absence of spin-orbit splitting in the initial $s$ state, significant dichroic contributions occur.

A successful ansatz to outline the physical origin of the SPEXAFS was based on a simple picture using a two-step model similar to the phenomenological attempt to describe of the near-edge XMCD. ${ }^{1,10,11}$ First, the absorption of circular polarized radiation in an unpolarized core state yields an outgoing photoelectron wave with a finite projection $\left\langle\sigma_{z}\right\rangle$ of its spin in the direction of the photon $k$ vector $\hat{z}$. This socalled Fano effect results from the interplay of the spin-orbit interaction in the initial and/or final state and the conservation of angular momentum. ${ }^{12}$ The spin polarization depends on the initial state: $\left\langle\sigma_{z}\right\rangle_{\left(L_{3}\right)}=0.25,\left\langle\sigma_{z}\right\rangle_{\left(L_{2}\right)}=-0.50$, $\left\langle\sigma_{z}\right\rangle_{\left(L_{1}\right)} \sim-0.15,\left\langle\sigma_{z}\right\rangle_{(K)} \sim+0.03$. For initial $p_{3 / 2}$ and $p_{1 / 2}$ spin-orbit split states the value of $\left\langle\sigma_{z}\right\rangle$ can be determined directly by vector coupling coefficients taking into account the constraint $\Delta m_{l}={ }_{(-)}^{+} 1$ for the transfer of the photoelectron into a $d$-like final state after absorption of a right (left) circular polarized photon. For $K$ or $L_{1}$ edges this procedure results in a value $\left\langle\sigma_{z}\right\rangle=0$. But a small spin-orbit splitting of the final $p$ states can, in principle, result in a nonvanishing photoelectron polarization even for $s_{1 / 2}$ initial states. In order to calculate $\left\langle\sigma_{z}\right\rangle$ for $s$ states one needs accurate dipole matrix elements for spin-up and spin-down transitions taking into account spin-orbit coupling in the final state, which is difficult to address. ${ }^{13}$ Therefore the given approximate values deduced from the difference of the transition strength are still the subject of discussions. In the second step the photoelectron wave then travels through the crystal and is scattered by the potentials of the neighboring atoms. If the sample is magnetized along the photon propagation direction, i.e., if the spin of its majoritylike electrons is aligned along the quantization axis $\hat{z}$, an exchange contribution in addition to the Coulomb scattering potential is acting on the backscattered, spin-polarized photoelectron wave. This interaction depends on the relative orientation of the magnetization to the spin of the photoelectron, which is determined by the helicity of the 
photon and the initial state as described above. Thus by forming the difference of the signal at opposite magnetic fields or at inverse photon helicities the SPEXAFS are obtained.

The aim of this paper is to develop the corresponding phenomenological equations for the SPEXAFS based on an additive exchange scattering term to the Coulomb scattering amplitude and to apply this formalism to data sets of the Co, Ni $K$ edges, and the Gd $L$ edges. New, highly precise dichroic spectra allow an accurate separation of the nextneighbor shell contributions in order to perform a parameter extraction for scattering at the next neighbors. As demonstrated in this paper high precision is essential to correct the data for magnetic multielectron excitations (MMEE's) in the absorption channel, ${ }^{14,4}$ which result in strong nonoscillatory contributions to the SPEXAFS in all three metals studied. These need to be subtracted prior to Fourier transformation. Since the excitations especially for the $K$ edges occur at low $k$ values covering a long energy range with a complex unknown structure, the correct reconstruction of the SPEXAFS contributions is difficult and possesses more uncertainties compared to the $L$ edges. This hampers especially the analysis of the corresponding iron $K$-SPEXAFS where the strongest MMEE with very complex energy variations are observed. Thus the Fe results, which have been obtained with high accuracy ${ }^{5}$ are not included in this presentation.

\section{EXPERIMENTAL DETAILS}

The experimental spectra have been recorded at the RöMo II bending magnet at the Hamburger Synchrotronstrahlungslabor using the two beam transmission mode. ${ }^{15}$ The estimated intensity of the photon beam monochromatized by a $\mathrm{Si}(111)$ double crystal was $10^{7-8}$ photons $/ \mathrm{sec} \mathrm{eV}$ with a degree of circular polarization of $60-70 \%$. The spectra were recorded alternating the direction of the external magnetic field of the solenoid, in which the metallic cold-rolled foils with thicknesses of about $2.5 \mathrm{mg} / \mathrm{cm}^{2}$ were mounted, every second at each energy. Several spectra with energy steps from $7 \mathrm{eV}$ in the pre-edge region, $1 \mathrm{eV}$ in the near-edge, and increasingly up to $5.6 \mathrm{eV}$ far from the edge were summed to improve statistics. Co and $\mathrm{Ni}$ were measured at room temperature, Gd was cooled down to $120 \mathrm{~K}$. The experiment determines the spin-averaged absorption coefficient $\mu_{0}(E)=1 / 2 \cdot\left[\mu^{+}(E)+\mu^{-}(E)\right]$ and the corresponding dichroic signal $\mu_{c}(E)=1 / 2 \cdot\left[\mu^{+}(E)-\mu^{-}(E)\right]$ for parallel $(+)$ and anti-parallel $(-)$ orientation of the photon "spin" with respect to the spin of the majoritylike electrons in the sample. The spin-averaged EXAFS spectra $\chi_{0}(k)=\left[\mu_{0}(k)-\mu_{\text {atom }}(k)\right] / \mu_{\text {atom }}(k)$ were deduced as a function of photoelectron wave number $k$ in the conventional way from the absorption profile by subtracting the free-atom absorption $\mu_{\text {atom }}$. The SPEXAFS spectra are denoted as the difference $\chi_{c}(k)=\left[\mu_{c}(k) / \mu_{\text {atom }}(k)\right] /\left[P_{c} \cdot M_{z}^{\prime}\right]$. They are rescaled for full circular polarization $P_{c}=1$ and complete alignment of the sample magnetization in the photon beam direction $M_{z}^{\prime}=1$ taking into account the tilt angle of $30^{\circ}$ with respect to the photon beam direction. For the Fourier transformation (FT) all spectra were weighted with $k^{1}$ and folded with a Kaiser-Bessel window. To extract the contribution of the next-neighbor shell the longest possible $k$ interval was used. For the comparison of the amplitudes in the FT spectra, identical intervals were chosen, which for $\mathrm{Gd}$ is determined by the energy difference between the $L_{2}$ and $L_{1}$ edge, i.e., $446 \mathrm{eV}$.

\section{THEORETICAL ASPECTS}

The conventional or spin-averaged EXAFS are described in terms of the Coulomb backscattering amplitude $f_{0}$ which depends on the scattering angle $\beta$ and the energy or wave vector $k$ of the photoelectron. In a ferromagnet the outgoing spin-polarized photoelectron emitted after the absorption of circularly polarized light experiences in addition an exchange interaction with the spin-polarized electrons carrying the atomic magnetic moment of the neighbors. Similar to the description of the magnetic Compton scattering for polarized photons by the Klein-Nishima formula ${ }^{16}$ we introduce an additional exchange scattering contribution $f_{c}$ to the overall scattering amplitude for parallel $(+)$ and antiparallel (-) orientation of the majoritylike spins to the photon-helicity. For a single electron-scattering process the amplitude becomes

$$
f(\beta, k)=f_{0}(\beta, k) \pm \sigma_{z} f_{c}(\beta, k),
$$

the two possible values for the spin of the photoelectron in the photon-beam direction $\hat{z}$ being $\sigma_{z}=1$ and $\sigma_{z}=-1$. Since the absorption experiment averages over many scattering processes with a particular ratio of spin-up and spin-down electrons one actually observes a mean spin polarization of the photoelectrons. We include this in our ansatz by replacing $\sigma_{z}$ by its mean value at the respective edge $\left\langle\sigma_{z}\right\rangle$ as given by the Fano factor. As the scattering angle $\beta$ is $\pi$ for single backscattering we will omit in the following the explicit angle dependence for simplicity and obtain:

$$
f(k)=f_{0}(k) \pm\left\langle\sigma_{z}\right\rangle f_{c}(k) .
$$

The electron mean free path $\lambda$, which is the inverse of the inelastic scattering cross section, might also display a dependence on the spin of the photoelectron:

$$
\lambda(k)=\lambda_{0}(k) \pm\left\langle\sigma_{z}\right\rangle \lambda_{c}(k) .
$$

All structural values, such as the coordination number $N$, the shell radius $r$, the Debye-Waller factor $D_{j}(k)=\exp \left(-2 \sigma^{2} k^{2}\right)$, representing the damping by lattice vibrations, are considered to be spin independent in this model. The amplitude reductions factor $S_{i}(k)$ is also assumed to be independent of spin, since its treatment is still a problem in the conventional EXAFS theory as well.

Using the conventional EXAFS formula ${ }^{17}$

$$
\begin{aligned}
\chi(k)= & -\sum_{j} \frac{N_{j}}{k r_{j}^{2}} S_{i}(k) D_{j}(k) e^{-2 r_{j} / \lambda_{0}(k)} \\
& \times \operatorname{Im}\left[e^{2 k r_{j}+i 2 \delta_{i}(k)} f(\beta, k)\right],
\end{aligned}
$$

one can construct $\chi^{+}$and $\chi^{-}$using Eqs. (2) and (3). The term $\delta_{i}$ describes the phase shift experienced by the photoelectron while leaving and returning to the absorbing atom. 
Neglecting second-order terms the spin-averaged EXAFS are given by

$$
\begin{aligned}
\chi_{0}(k)= & \frac{1}{2}\left[\chi^{+}(k)+\chi^{-}(k)\right] \\
= & -\sum_{j} \frac{N_{j}}{k r_{j}^{2}} S_{i}(k) D_{j}(k) e^{-2 r_{j} / \lambda_{0}(k)} \\
& \times F_{0, j}(k) \sin \left(2 k r+2 \delta_{i}(k)+\Phi_{0, j}(k)\right) .
\end{aligned}
$$

This corresponds to the well-known conventional EXAFS formula in which the complex scattering amplitude was replaced by its amplitude $F_{0, j}(k)$ and phase $\Phi_{0, j}(k)$ with $f_{0, j}(k)=F_{0, j}(k) \exp \left(i \Phi_{0, j}(k)\right)$.

The corresponding equation for the SPEXAFS can be written as

$$
\begin{aligned}
\chi_{c}(k)= & \frac{1}{2}\left[\chi^{+}(k)-\chi^{-}(k)\right] \\
= & -\sum_{j} \frac{N_{j}}{k r_{j}^{2}} S_{i}(k) D_{j}(k) e^{-2 r_{j} / \lambda_{0}}\left\langle\sigma_{z}\right\rangle \\
& \times\left\{F_{c, j}(k) \sin \left(2 k r+2 \delta_{i}(k)+\Phi_{c, j}(k)\right)\right. \\
& \left.+\frac{2 r_{j} \lambda_{c}(k)}{\lambda_{0}(k)^{2}} F_{0, j}(k) \sin \left(2 k r+2 \delta_{i}(k)+\Phi_{0, j}(k)\right)\right\},
\end{aligned}
$$

the complex scattering amplitudes again replaced by their amplitude and phase. If the absorbing atom carries a spin moment as well, the outgoing photoelectron will be influenced by the exchange interaction also upon leaving the atom adding a magnetic contribution $\delta_{c, i}$ to $\delta_{i}(k)$. Since the numerical procedure does not allow the separation of a magnetic contribution to $\delta_{i}(k)$ from $\Phi_{c, j}(k)$, the entire spin dependence of the photoelectron's phase has been included in the value of $\Phi_{c, j}(k)=\delta_{c, i}+\phi_{c, j}$. It is interesting to note that the SPEXAFS function includes a term proportional to $\lambda_{c}(k) \cdot F_{0, j}(k)$. This enables one to make an estimation of the spin-dependent mean free path simply by identifying contributions in the SPEXAFS spectrum which scale with $F_{0, j}$.

For simplicity a few phenomenological clues about the shape and character of the spin-dependent quantities are introduced. Evidently the effective exchange interaction results from those electrons that are carrying the magnetic moment, i.e., the $3 d$ electrons in transition metals (TM's) and the $4 f$ electrons in the rare earth (RE). Hence the shape of the scattering potential should be comparable to that of a single electronic shell. As pointed out by Teo ${ }^{17}$ the Coulomb scattering amplitude for simple potentials $(Z \leqslant 35)$ can by approximated by a Lorentzian and the phase by a polynomial. In the same manner the electron mean free path can be substituted as $\lambda_{0}(k)=k^{n} / \eta$ where $n=1$ or 2 . Since the number of magnetic electrons is small we adopt this view and use a Lorentzian for the spin-dependent backscattering amplitude and a polynomial of degree 3 for the phase shift. For the spindependent mean free paths we used a straight line
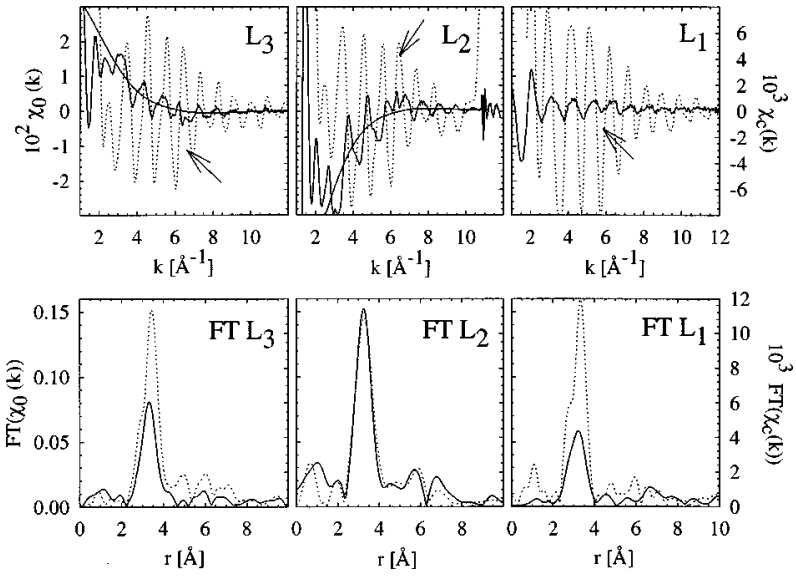

FIG. 1. The spin-averaged $\chi_{0}(k)($ dots $)$ and spin-dependent (solid) $\chi_{c}(k)$ spectra at the $L_{1,2,3}$ edges of Gd metal. The corresponding Fourier transforms after subtraction of the magnetic background [solid line in the $\chi_{c}(k)$ spectra] are shown in the lower panel. The arrows mark the location of multielectron transitions.

$$
\begin{gathered}
F_{c, j}(k)=\frac{A}{1+\left(1 / B^{2}\right)(k-C)^{2}}, \\
\Phi_{c, j}(k)=p_{3} k^{3}+p_{2} k^{2}+p_{1} k+p_{0}, \\
\lambda_{c}(k)=\xi \cdot k .
\end{gathered}
$$

Note that in Eq. (7a) $A$ represents the amplitude, the factor $B^{2}$ corresponds to the full width at half maximum (FWHM), and $C$ gives the location of the maximum amplitude. Using Eqs. (6) and (7) it is possible to extract the $k$ distributions of the magnetic backscattering amplitude and phase shift from the experimental data. This is accomplished in three steps: (1) the values $F_{0, j}(k), \Phi_{0, j}(k), \delta_{i}(k), \lambda_{0}(k)$ are calculated using the FEFF code of Rehr, ${ }^{18}$ (2) the factors $D_{j}$ and $S_{i}$ together with the energy reference $E_{0}$ (i.e., the difference of the kinetic energy origin of the photoelectron between the theoretical calculation and the inflection point of the absorption profile) are determined by fitting the $\chi_{0}(k)$ spectra; and finally (3) with the magnetic backscattering amplitude $F_{c, j}(k)$ and phase $\Phi_{c, j}(k)$ as well as $\lambda_{c}(k)$ the remaining free parameters are deduced from the $\chi_{c}(k)$ profiles. The second step is useful to reduce the number of free parameters in the overall fitting procedure.

\section{RESULTS AND DISCUSSION}

\section{A. General overview}

The experimental results of the spin-averaged $\left[\chi_{0}(k)\right]$ and spin-dependent EXAFS $\left[\chi_{c}(k)\right]$ measurements at the $L$ edges of $\mathrm{Gd}$ and the $\mathrm{Co}$ and $\mathrm{Ni} K$ edge and their Fourier transforms are shown in Figs. 1 and 2. In general the observed frequencies in the EXAFS and SPEXAFS signals are very similar as expected for pure systems where the magnetic and electronic neighborhood are identical. While the SPEXAFS oscillate around zero at the $K$ edges of $\mathrm{Co}$ and $\mathrm{Ni}$ and the $\mathrm{Gd} L_{1}$ edge, a strong magnetic background occurs at the $L_{2}$ and $L_{3}$ edge of $\mathrm{Gd}$, as indicated by the solid line in 

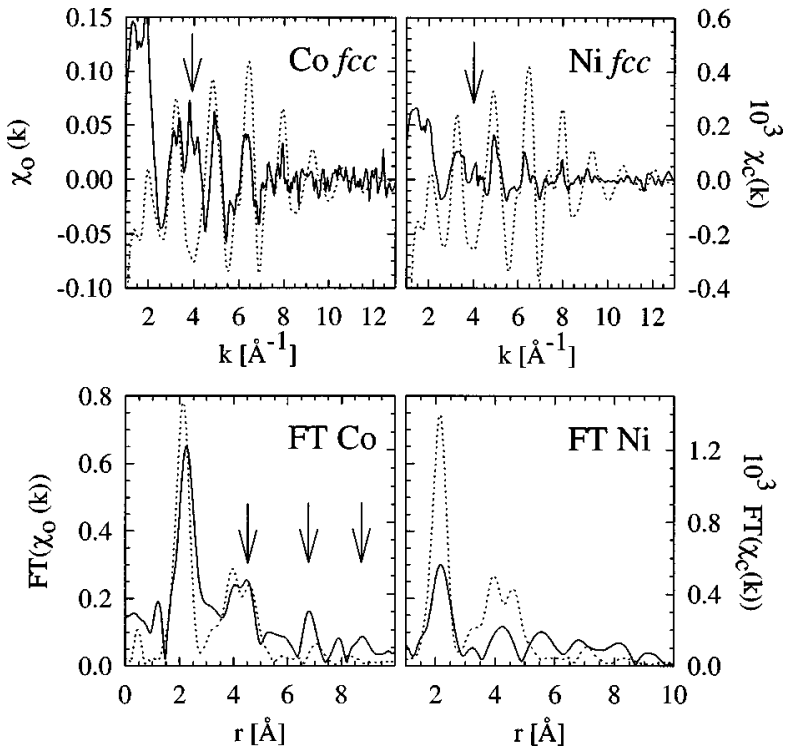

FIG. 2. The spin-averaged $\chi_{0}(k)$ (dots) and spin-dependent $\chi_{c}(k)$ spectra (solid) of the Co and Ni $K$ edge (top). The locations of multielectron transitions is marked by the arrows. The corresponding Fourier transforms are shown below with contributions of multiple path lines in Co pointed by the arrows.

Fig. 1, which displays the same ratio of -2 in amplitude between the two edges as the magnetic EXAFS and the corresponding near-edge XMCD. The occurrence of a similar smooth background in the normal EXAFS was discussed by Rehr et al. ${ }^{19}$ The observed dichroic contribution here, increasing approaching the origin of the energy scale, can be viewed as a very low-frequency oscillation, originating from the scattering from the spin density of polarized $d$ states at the fringes of the absorbing atom carrying a magnetic moment in case of Gd of about $0.5 \mu_{\mathrm{B}} \cdot{ }^{20}$ For the further analysis this background as indicated was subtracted from the dichroic spectra.

All SPEXAFS are also superimposed by additional highfrequency contributions resulting in a significantly sharper fine structure of the dichroic part. We attribute this observation to a contribution of strongly enhanced magnetic multiple-scattering paths in the case of a spin-polarized photoelectron traveling through the ferromagnet. The effect can be confirmed in the Fourier transform of the SPEXAFS, well seen for Co, where lines in FT $\left[\chi_{c}(k)\right]$, as marked by the arrows in the lower part of Fig. 2, at twice and more times the distance of the first shell are clearly larger in amplitude compared to multiple-path lines in the corresponding spinaveraged EXAFS FT.

A second significant difference is found at characteristic energies where the regular oscillations in the SPEXAFS are superimposed by an additional structure as pointed out by the arrows in the $\chi_{c}$ spectra. These features are attributed to strong magnetic multi-electron excitations (MMEE), where the surplus of the photon energy is used to excite a second electron. ${ }^{14}$ Since these excitations display a the strong magnetic character the second electron must be transfered into a valence state which is strongly spin and/or orbitally polarized. Possible transitions for the $L_{2 / 3}$ edge are $2 p 4 d \rightarrow(5 d)^{2}$ and $2 p 4 d \rightarrow 6 p 4 f$, for the $L_{1}$ edge $2 s 4 d \rightarrow 6 p 5 d$. At the $K$
TABLE I. Parameters of the spin-dependent backscattering amplitude and phase for the Gd $L$ edges following Eq. (7). The error resulting from the fitting procedure and the uncertainty of the data is given in brackets as change in the last digit.

\begin{tabular}{lccl}
\hline \hline Parameter & \multicolumn{1}{c}{$\mathrm{L}_{1}$} & \multicolumn{1}{c}{$\mathrm{L}_{2}$} & \multicolumn{1}{c}{$\mathrm{L}_{3}$} \\
\hline$A$ & $1.70(1) \times 10^{-2}$ & $4.83(1) \times 10^{-2}$ & $2.43(1) \times 10^{-2}$ \\
$B^{2}$ & $10.6(5)$ & $10.6(5)$ & $11.7(6)$ \\
$C$ & $5.5(1)$ & $4.1(1)$ & $4.5(1)$ \\
$p_{0}$ & $4.5(1)$ & $5.6(1)$ & $9.1(1)$ \\
$p_{1}$ & $-2.8(1)$ & $-3.6(1)$ & $-3.8(1)$ \\
$p_{2}$ & $0.29(1)$ & $0.41(1)$ & $0.43(1)$ \\
$p_{3}$ & $7.5(1) \times 10^{-3}$ & $1.5(1) \times 10^{-2}$ & $1.5(1) \times 10^{-2}$ \\
$\xi$ & $0.00(1)$ & $0.00(1)$ & $0.00(1)$ \\
\hline \hline
\end{tabular}

edge the second electron can be excited from a $3 s$ or $4 p$ state, so that the MMEE profile reflects a linear combination of the $s-p$ or $-d$ spin/orbital density of states at $E_{f}$. The presence of these additional excitation lines influences the analysis by introducing unphysical structures at low $r$ values and modifying the line intensities of the Fourier transform. ${ }^{21}$ The correction of these features is still an open problem, especially for the $K$-edge SPEXAFS where these oscillations occur close to the absorption edge with a very detailed broad structure. Therefore the rather large difference in shape at about $3 \AA$ in the FT spectra of Co, which strongly depends on the estimated MMEE contribution subtracted from the data, might indicate that the approximated polynomial taken up to now to describe the MMEE is still too simple. The line shape for the MMEE at the $\mathrm{Gd} L$ edges is much simpler and is well represented by a polynomial. A thorough discussion of the magnetic multielectron effects will be subject to a forthcoming publication. In the following section we discuss the data analysis results separately for the ferromagnetic systems applying the method described above.

\section{B. Gd metal}

Applying Eqs. (5) and (6) to the Gd-SPEXAFS presented in Fig. 1 and using the parametrization Eq. (7) we were able to deduce the spin-dependent scattering amplitudes and phases for the first-neighbor contributions at the Gd $L$ edges. The numeric results are given in Table I and the graphs plotted in Fig. 3. The Coulomb scattering amplitude presented in Fig. 3 by the dashed line for $\mathrm{Gd}$ shows three prominent peaks at about $1.5,6$, and $13 \AA^{-1}$ which are caused by the more complex electron configuration in the heavier atoms as the rare earth. They are normally referred to as RamsauerTownsend resonances. ${ }^{22}$ The enhanced first peak in $F_{0, j}(k)$ at $1.5 \AA^{-1}$ at the $L_{1}$ edge is caused by the different angular symmetry of the electron wave. This is of only minor importance for the EXAFS analysis since the intervals of evidence for the Fourier transform have a minimum $k$ value of about $2.5 \AA^{-1}$. The best agreement between the measured and fitted SPEXAFS, was achieved by using only one Lorentzian for the magnetic-scattering amplitude. With the maximum of $F_{c, j}(k)$ located at $5.5 \AA^{-1}\left(L_{1}\right), 4.1 \AA^{-1}\left(L_{2}\right)$, and $4.5 \AA^{-1}$ $\left(L_{3}\right)$ it is positioned close to the second peak of $F_{0, j}(k)$, the 


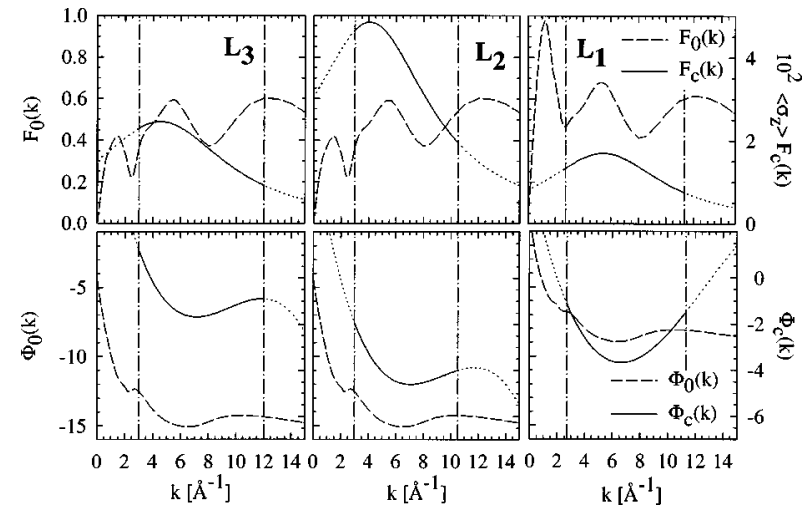

FIG. 3. The spin-averaged (dots) and spin-dependent (solid) scattering amplitudes and phases of Gd hcp metal at the $L$ edges for the first-neighbor shell. The vertical lines represent the actual data interval used in the fitting procedure.

FWHM is about $11 \AA^{-1}$ within statistical accuracy. The magnetic phase shifts at the $\mathrm{Gd} L$ edges are quite similar in their shape. They are about a factor 5 smaller, but resemble approximately the form of the spin-averaged value $\Phi_{0, j}(k)$. There is a shift of $\pi$ between the $L_{1}, L_{2}$ compared to the $L_{3}$ edge. A magnetic contribution to the electron mean free path was not found for $\mathrm{Gd}$ within the statistical uncertainties of about $1 \%$ in $\chi_{c}(k)$.

A comparison of the amplitude of the first maximum in the $L_{1,2,3}$ SPEXAFS FT and the $F_{c, j}(k)$ values indicates that the dichroic contribution scales definitely with $\left\langle\sigma_{z}\right\rangle$. The Fano effect predicts a factor of -2 in the spin polarizations of the $L_{2}$ and $L_{3}$ edge. This is exactly the result of the parameter extraction, which yields a factor of 2 between the magnetic-scattering amplitudes $F_{c, j}(k)$. The sign can be determined from the phases $\Phi_{c, j}(k)$ at the $L_{2}$ and $L_{3}$ edge which are exactly separated by a constant offset $\pi$, thus giving a minus sign to the relation of the spin polarization. Using the opposite procedure we are able to estimate the value and the sign of the spin polarization $\left\langle\sigma_{z}\right\rangle$ at the $L_{1}$ edge. From the relation of the amplitude maxima at the $L_{1}$ and $L_{2}$ edge scaled by $\left\langle\sigma_{z}\right\rangle_{L_{2}}$ one obtains a value of $17 \%$. Since the amount of phase shift is about that of the $L_{2}$ edge, we get a negative polarization of $\left\langle\sigma_{z}\right\rangle_{\left(L_{1}\right)}=-17 \%$ in exact agreement with an estimative theoretical calculations of the $2 s_{1 / 2} \rightarrow p_{1 / 2}$ and $2 s_{1 / 2} \rightarrow p_{3 / 2}$ transition matrix elements ${ }^{23}$ and XMCD studies at the Gd $L_{1}$ near-edge region.

To get a hint what kind of electrons contribute to the backscattering potential in the $k$ range of $3-10.5 \AA^{-1}$ the Coulomb backscattering amplitudes for the $4 f$ elements La, $\mathrm{Pr}, \mathrm{Gd}, \mathrm{Ho}, \mathrm{Tm}$ are calculated as shown in Fig. 4. Ascending through the rare-earth series the broad maximum at about 12 $\AA$ is shifted towards higher energies as result of the lanthanide contraction, while the amplitude of the peak remains almost constant. The dominant quantitative change in $F_{0, j}(k)$ occurs in the $k$ region between 2.5 and $7 \AA^{-1}$ where the amplitude is strongly decreasing with increasing $Z$. Since the $4 f$ shell is filled while ascending through the rare-earth series this middle peak must be related to the part of the Coulomb potential formed predominantly by the $4 f$ shell. The magnetic-scattering amplitude has its maximum exactly in the $k$ range where the photoelectron interacts mostly with the

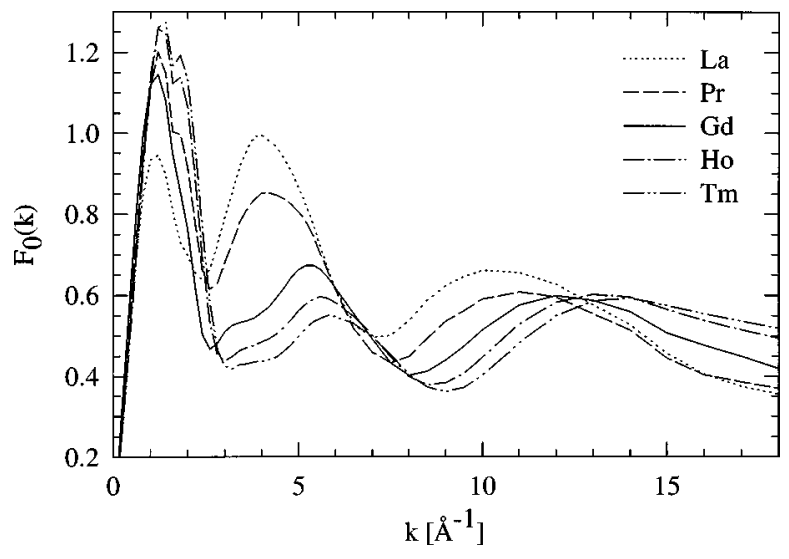

FIG. 4. Backscattering amplitude $F_{0,1}(k)$ for the first-neighbor shell of selected RE metals. Besides the shift of the maximum of the right peak towards higher energies with increasing $Z$, the change in the middle peak is clearly related to the filling of the $4 f$ shell.

magnetic $4 f$ electrons. The $k$ distribution of $F_{c, j}(k)$ found by our fitting procedure reflects directly that the physical origin of the magnetic EXAFS can be related to the exchange effect of the scattering of the photoelectron at the polarized $4 f$ electrons. The smaller width in the $k$ distribution explains directly the fact that only the seven distinguished $4 f$ electrons are involved in the magnetic scattering.

In analogy to the conventional EXAFS analysis, we have used our extracted backscattering amplitude and phase to reconstruct the measured spectrum using single scattering paths only. The result is presented in Fig. 5. The overall agreement is reasonably good. On the other hand, it is obvi-

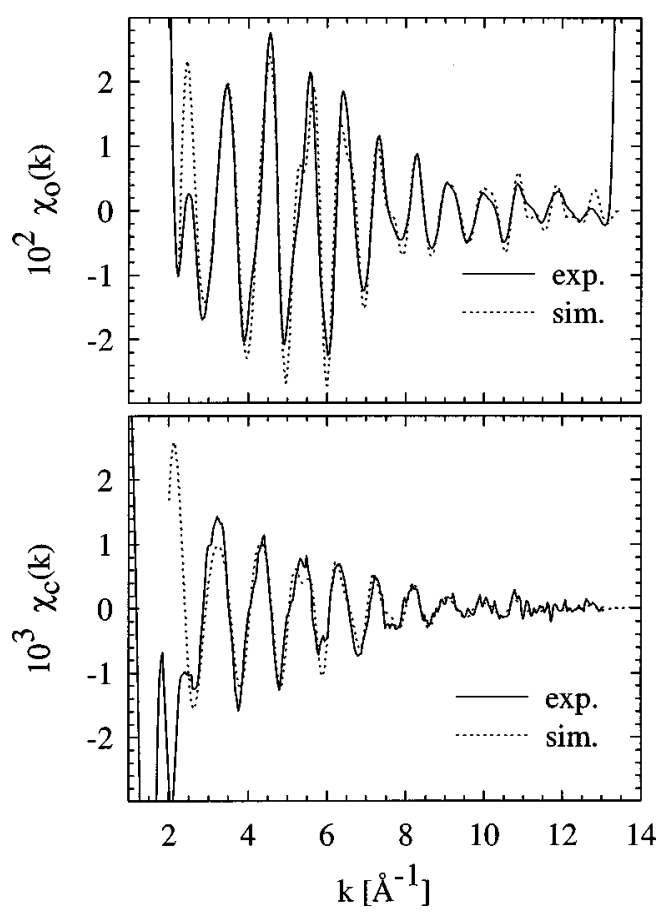

FIG. 5. Reconstruction of the measured Gd $L_{3}$ EXAFS (top) and SPEXAFS (bottom) signal using the extracted spin-dependent backscattering amplitude and phase. Four shells were used for the modeling. 
TABLE II. Corresponding parameters of the spin-dependent backscattering amplitude and phase of $\mathrm{Co}$ and $\mathrm{Ni}$. The error is given in brackets as change in the last digit.

\begin{tabular}{lll}
\hline \hline Parameter & \multicolumn{1}{c}{ Co fcc } & \multicolumn{1}{c}{$\mathrm{Ni} \mathrm{fcc}$} \\
\hline$A$ & $1.77(3) \times 10^{-3}$ & $0.78(3) \times 10^{-3}$ \\
$B$ & $4.4(4)$ & $5.2(4)$ \\
$C$ & $5.2(1)$ & $4.9(1)$ \\
$p_{0}$ & $2.5(1)$ & $2.5(1)$ \\
$p_{1}$ & $-0.58(1)$ \\
$p_{2}$ & $-0.52(1)$ & $2.3(1) \times 10^{-2}$ \\
$p_{3}$ & $1.5(1) \times 10^{-2}$ & $1.2(1) \times 10^{-3}$ \\
$\xi$ & $1.6(1) \times 10^{-3}$ & $0.00(1)$ \\
\hline \hline
\end{tabular}

ous that also in the SPEXAFS multiple-scattering contributions need to be considered in order to fully reproduce the data.

\section{Co and Ni metal}

The magnetic backscattering amplitudes and phases for $\mathrm{Co}$ and Ni as calculated from the extracted parameters given in Table II are presented in Fig. 6. The Coulomb backscattering amplitude exhibits a very broad $k$ distribution and peaks for $\mathrm{Co}$ at about $6 \AA^{-1}$. As found at the $L$ edges of $\mathrm{Gd}$ its magnetic counterpart has a much narrower distribution in $k$ space. The maximum located at lower $k$ values at $5.2 \AA^{-1}$ with an amplitude of $1.8 \times 10^{-3}$ and a width of $4.4 \AA^{-1}$ (FWHM). For Ni the maximum of $F_{0, j}(k)$ is slightly smaller and shifted towards higher energies, a consequence of the stronger Coulomb potential correlated to a smaller atomic radius. Again the amplitude of the magnetic counterpart is shifted towards lower $k$ values at $4.9 \AA^{-1}$ with an amplitude of $0.78 \times 10^{-3}$ and displays the same narrow $k$ distribution $\left(5.2 \AA^{-1}\right)$ as Co. The spin-dependent phase shifts are very similar for $\mathrm{Co}$ and $\mathrm{Ni}$. They are smaller, but follow approximately the shape of $\Phi_{0, j}(k)$. As for the Gd $L$ edges a mag-

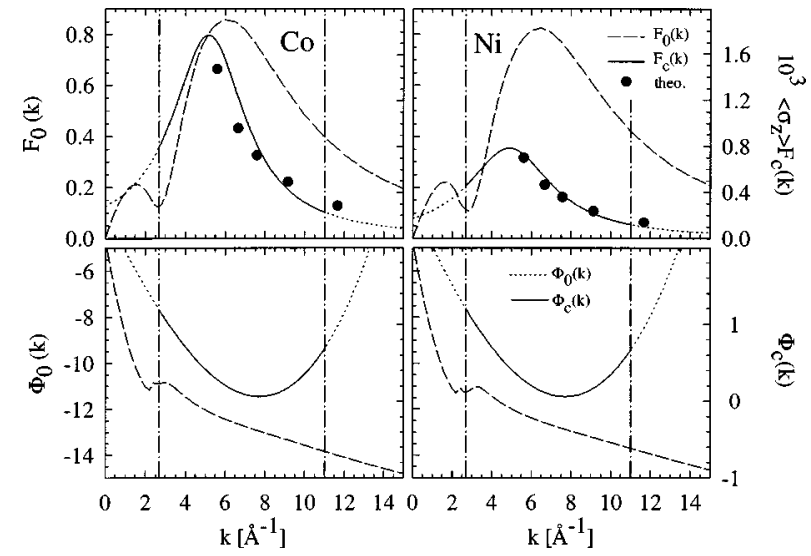

FIG. 6. The spin-averaged (dots) and spin-dependent (solid) scattering amplitudes and phases of $\mathrm{Co}$ fcc and $\mathrm{Ni}$ fcc metal from $K$ edge absorption for the first-neighbor shell. The vertical lines represent the actual data interval used in the fitting procedure. The points show a calculation of Ref. 25 for polarized electron exchange scattering from oriented atoms in the Born-Ockhur approximation.

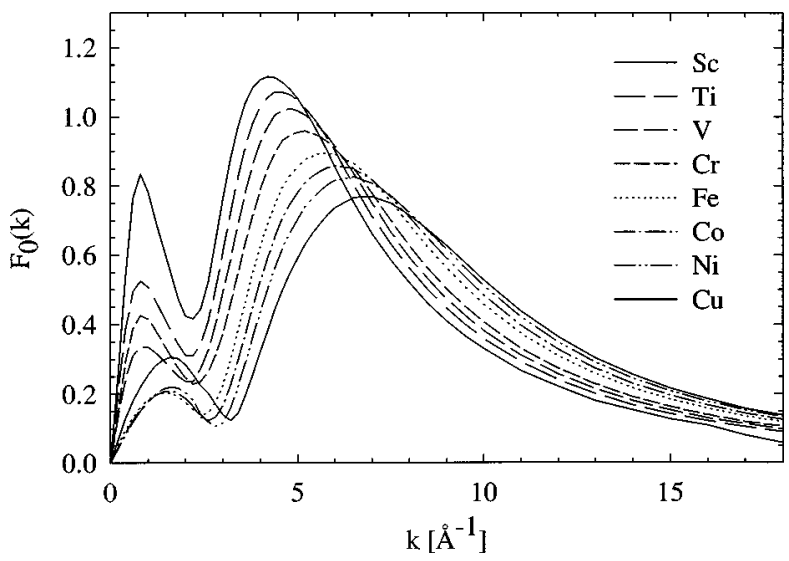

FIG. 7. Backscattering amplitude $F_{0,1}(k)$ for the first-neighbor shell of selected transition metals. The scattering amplitude shifts towards higher energies with increasing $Z$ while its peak value decreases.

netic contribution to the electron mean free path was found for $\mathrm{Co}$ and $\mathrm{Ni}$ to be smaller than $1 \%$.

While the spin-dependent-scattering amplitude for $\mathrm{Gd}$ peaked at the second maximum of the Coulomb scattering amplitude with a rather broad distribution, the maximum of $F_{c, j}(k)$ for Co and $\mathrm{Ni}$ occurs at considerably lower $k$ values than for the Coulomb scattering and a narrower distribution. Since the magnetic moment of $\mathrm{Co}$ and $\mathrm{Ni}$ is established by the exchange splitting of the electrons in the outer $3 d$ state, this behavior can be well understood. It reflects directly the more delocalized character of the electronic states which carry the magnetic moment of the $3 d$ transition elements. A comparison of the $F_{0, j}(k)$ function for several $3 d$ elements as shown in Fig. 7 proves that indeed the $k$ region, where the spin-dependent backscattering part contributes the dominate change in the $F_{0, j}(k)$ amplitude, is found.

The results for these metals can be compared with a theoretical approach to calculate exchange phenomena in the electron-atom scattering process based on the Born-Ockhur approximation given by Matthew. ${ }^{25}$ In this model the exchange contribution to the conventional Born approximation is related to the simple Born amplitude via a Fourier expansion. The calculation is done for forward scattering, but it is estimated that the results for backscattering should be somewhat smaller in its $k$ distribution, but exhibit a similar energy dependence. In the high-energy region it is shown that the spin-dependent scattering amplitude scales approximately with $k^{-2}$ for energies above $150 \mathrm{eV}$. The results of the calculation of the exchange scattering amplitude are shown as dots in Fig. 6. The agreement between the two results is excellent, the scattering amplitude possesses the same shape and amplitude. Furthermore a ratio of $10^{-2}$ between the inelastic and elastic contributions to the exchange scattering amplitude is predicted in this theoretical approximation resulting in negligible inelastic exchange scattering effects. This is in agreement with the results found from our simple model, indicating that the spin-dependent part to the mean free path is by 2 orders of magnitude smaller compared to the elastic exchange effect. A reconstruction of the full spectrum with the extracted amplitudes and phases taking into 


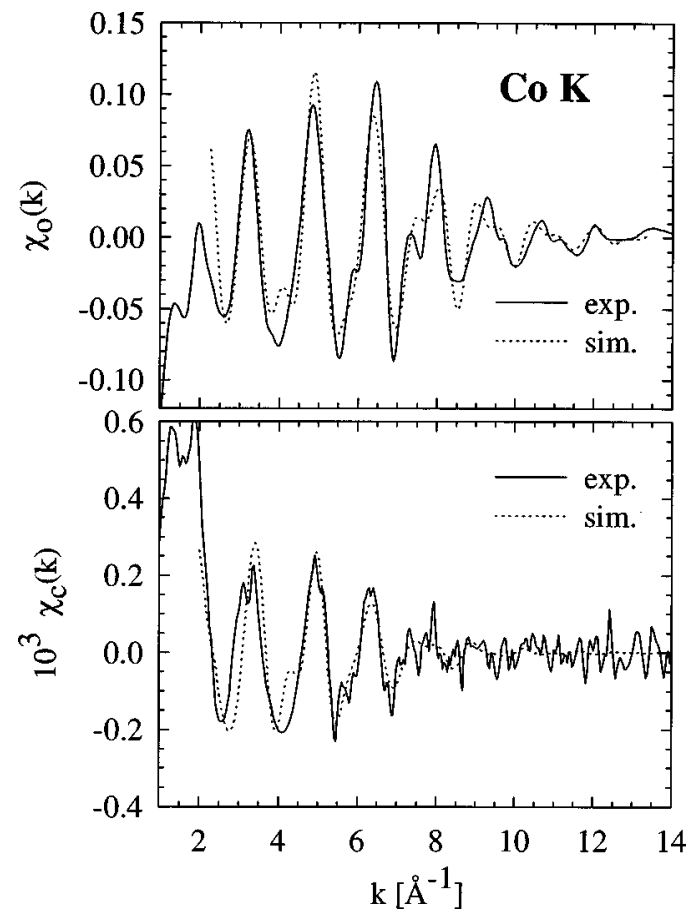

FIG. 8. Reconstruction of the measured Co $K$ EXAFS (top) and SPEXAFS (bottom) signal using the extracted spin-dependent backscattering amplitude and phase. Four shells were used for the modeling.

account the first four coordination shells is shown in Fig. 8. As in Gd a good agreement between experiment and simulation is observed.

As shown in earlier systematic studies the SPEXAFS signal is found to be correlated to the magnetic spin moment $\mu_{\mathrm{sp}}$ of the scattering neighbor times the photoelectron polarization. ${ }^{24}$ This is most easily seen by comparing the maxima of the Fourier transform for which this relation can be written as

$$
\frac{1}{\left\langle\sigma_{z}\right\rangle} \frac{\max \left[\mathrm{FT}\left(\chi_{c}(k)\right)\right]}{\max \left[\mathrm{FT}\left(\chi_{0}(k)\right)\right]}=+2.4(2) \% \mu_{\mathrm{S}}\left[\mu_{\mathrm{B}}\right] .
$$

Thus one expects that the ratio of the extracted exchange and Coulomb backscattering amplitudes rescaled by the photoelectron polarization shows the same dependence of the magnetic spin moment.

Forming the relation between the maxima of the magnetic-scattering amplitudes of $\mathrm{Co}$ and $\mathrm{Ni}$ one receives the number 2.3. The ratio of the atomic spin moments $\mu_{\mathrm{sp}}(\mathrm{Co}) / \mu_{\mathrm{sp}}(\mathrm{Ni})=2.5$ is somewhat larger. That the value $F_{c, \text { max }}(\mathrm{Co}) / F_{c, \text { max }}(\mathrm{Ni})$ tends to be by $10 \%$ lower can well be explained by two arguments. First the spin polarization at the $\mathrm{Ni} K$ edge is expected to be about $10 \%$ higher than at the Co $K$ edge $^{23}$ since the larger $Z$ yields a higher relativistic spinorbit effect from which the spin polarization originates. Second, due to the lower $Z$ value Co has a somewhat enhanced fraction of delocalized magnetic electrons compared to $\mathrm{Ni}$, which would contribute to $F_{c}$ in a lower $k$ region, where the data is not accurate enough.

Knowing about the shape and value of the backscattering amplitude we want to give at least a hint on the problem of the enhanced multiple-scattering path lines. Since the scat- tering amplitudes are significantly different for spin-up and spin-down electrons, the fraction of the majoritylike photoelectron increases with each scattering process, while that of the minoritylike photoelectron decreases, thus enhancing the overall spin polarization along the scattering path and therefore the contribution of this path to the dichroic signal. Before returning to the absorbing atom an electron is scattered $n$ times with a scattering amplitude $F^{\uparrow \downarrow} \approx F_{0}^{n}\left[1 \pm n\left(F_{c} / F_{0}\right)\right]$. Since for Co, as an example $F_{c} / F_{0}=2.4 \% \cdot 1.6 \sim 4 \%$, the spin polarization is increased by a factor approximately 2(3) in the first (second) scattering process giving rise to an increasing magnetic-scattering effect related to the fine structure in the SPEXAFS as seen in Figs. 5 and 8. A detailed discussion of this phenomenon is the subject of a forthcoming publication.

\section{CONCLUSION}

In summary, we have presented a phenomenological model of the SPEXAFS which is based on the assumption that the exchange effects can be described as an additive contribution to the Coulomb scattering amplitude. From a parameter extraction procedure magnetic backscattering amplitudes as a function of photon energy at the Co and $\mathrm{Ni} K$ edge and the Gd $L$ edges have been derived. Although, in principle, the picture is oversimplified to describe the complex phenomenon of the magnetic EXAFS in detail the systematics observed in the experimental results indicate that on this level their physical origin and the observed systematics can be well understood. It explains straightforwardly that the SPEXAFS are proportional to the spin polarization of the photoelectron and that the relative strength of the SPEXAFS scales with the magnetic moment of the neighboring atoms. The $k$ distribution of $F_{c, j}(k)$ reflecting the radial distribution of the magnetic electrons is found exactly in the region, where the magnetic shell contributes predominantly to the scattering potential as seen from a comparison of the backscattering amplitudes for various $3 d$ and $4 f$ atoms. It is in excellent agreement with theoretical values predicted by the Born-Ockhur approximation.

The presented results promise that highly accurate studies of magnetic EXAFS provide-beside the element-specific determination of the magnetic short-range order-a new possibility to determine electron-atom exchange scattering cross sections inside the solid, free of spin orbit and surface effects, creating an important basis to test the theory of spin scattering. We expect that by further improvement of the accuracy of the data and with a better insight into the strong MMEE and enhanced multiple-scattering contributions, the exchange scattering cross section even for much lower electron energies can be addressed by this spectroscopic method.

\section{ACKNOWLEDGMENTS}

We would like to thank J. J. Rehr, A. Ankudinov, H. Ebert, and M. Knülle for stimulating discussions. The experimental help of the technical staffs at HASYLAB, the TU Munich, and the University of Augsburg is highly appreciated. This work is supported by the German Federal Minister of Education and Research and the Deutsche Forschungsgemeinschaft. 
${ }^{1}$ G. Schütz, W. Wagner, W. Wilhelm, P. Kienle, R. Zeller, R. Frahm, and G. Materlik, Phys. Rev. Lett. 58, 737 (1987).

${ }^{2}$ G. Schütz, R. Frahm, P. Mautner, R. Wienke, W. Wagner, W. Wilhelm, and P. Kienle, Phys. Rev. Lett. 62, 2620 (1989).

${ }^{3}$ V. Chakarian, Y. U. Idzerda, G. Meigs, and C. T. Chen, IEEE Trans. Magn. 31, 3307 (1995).

${ }^{4}$ D. Ahlers and G. Schütz, J. Phys. IV 7, C2-457 (1997); 7, C2-59 (1997), and references therein.

${ }^{5}$ G. Schütz and D. Ahlers, in Spin-Orbit Influenced Spectroscopies of Magnetic Solids, edited by H. Ebert and G. Schütz, Lecture Notes in Physics Vol. 466 (Springer, Heidelberg, 1996).

${ }^{6}$ M. Knülle, D. Ahlers, and G. Schütz, Solid State Commun. 94, 267 (1995).

${ }^{7}$ A. Ankudinov and J. J Rehr, Phys. Rev. B 52, 10214 (1995).

${ }^{8}$ H. Ebert, V. Popescu, and D. Ahlers, J. Phys. IV 7, C2-131 (1997)

${ }^{9}$ C. Brouder, M. Alouani, C. Giorgetti, E. Dartyge, and F. Baudelet, in Spin-Orbit Influenced Spectroscopies of Magnetic Solids (Ref. 5).

${ }^{10}$ G. Schütz, M. Knülle, R. Wienke, W. Wilhelm, W. Wagner, P. Kienle, and R. Frahm, Z. Phys. B 73, 67 (1988).

${ }^{11}$ G. Schütz, R. Wienke, W. Wilhelm, W. Wagner, P. Kienle, R. Zeller, and R. Frahm, Z. Phys. B 75, 495 (1989).
${ }^{12}$ U. Fano, Phys. Rev. 178, 131 (1969).

${ }^{13}$ S. Stähler, G. Schütz, and H. Ebert, Phys. Rev. B 47, 818 (1993).

${ }^{14}$ E. Dartyge, A. Fontaine, C. Giorgetti, S. Pizzini, F. Baudelet, G. Krill, C. Brouder, and J. P. Kappler, Phys. Rev. B 46, 3155 (1992).

${ }^{15}$ G. Schütz, R. Frahm, R. Wienke, W. Wilhelm, W. Wagner, and P. Kienle, Rev. Sci. Instrum. 60, 1661 (1989).

${ }^{16}$ R. M. Steffen and H. Frauenfelder, in Alpha-, Beta- and Gamma ray Spectroskopy, edited by K. Siegbahn (North-Holland, Amsterdam, 1968), Vol. 2, p. 1456.

${ }^{17}$ B. K. Teo, EXAFS: Basic Principles and Data Analysis (Springer, Berlin, 1986).

${ }^{18}$ J. J. Rehr, Jpn. J. Appl. Phys., Part 1 32, 8 (1993).

${ }^{19}$ J. J. Rehr, C. H. Booth, F. Bridges, and S. I. Zabinsky, Phys. Rev. B 49, 12347 (1994).

${ }^{20}$ J. Sticht and J. Kübler, Solid State Commun. 53, 529 (1985).

${ }^{21}$ J. Chaboy, A. Marcelli, and T. A. Tyson, Phys. Rev. B 49, 11652 (1994).

${ }^{22}$ B. Lengeler, J. Phys. (Paris), Colloq. 47, C8-75 (1986).

${ }^{23}$ H. C. Pauli and V. Raff, Comput. Phys. Commun. 9, 392 (1975).

${ }^{24}$ G. Schütz, M. Knülle, and H. Ebert, Phys. Scr. T49, 302 (1993).

${ }^{25}$ J. A. D. Matthew, Phys. Rev. B 25, 3326 (1982). 